\title{
Analytical Solution of Laminar Boundary Layer Equations Over A Flat Plate by Homotopy Perturbation Method
}

\author{
A. Majidian, M. khaki, M. khazayinejad \\ Islamic Azad University, Sari Branch, Sari, Iran \\ E-mail: m.khazayinejad@gmail.com
}

\begin{abstract}
In this paper, the problem of an incompressible viscous flow over a flat plate is presented. The Homotopy perturbation method (HPM) is employed to solve the well-known Blasius nonlinear differential equation. It has been tried to use a special technique by which one be able to obtain solutions that are very close to the exact solution of the equation. The obtained results have been compared with the exact solution of Blasius equation and another results obtained in previous works so that the high accuracy of results are clear. Also it is found that this method is powerful mathematical tool and can be applied to a large class of linear and nonlinear problems in different fields of science and engineering especially some fluid mechanics equations.
\end{abstract}

Keywords: Boundary layer, Nonlinear equations, Blasius equation, Howarth number, Homotopy perturbation method (HPM)

\section{Introduction}

The governing equations the most physical phenomena are non-linear. Except in a limited number of these problems, we have difficulty in finding their exact analytical solutions and others must be solved by other methods. Therefore, approximate analytical solutions are searched were introduced approximate. Analytical methods have always been of interest to scientists. Perturbation method is one of the well-known methods to solve nonlinear problems; it is based on the existence of small/large parameters, the so-called perturbation quantity [1, 2]. Many nonlinear problems do not contain such kind of perturbation quantity, and 
we can use non-perturbation methods, such as the $\delta$-expansion method [3], the artificial small parameter method [4], the Adomian's decomposition method [5], the Variational iteration method (VIM) [6], and the Homotopy analysis method (HAM) [7].

One of the strong analytical methods for eliminating small parameter is applying Homotopy perturbation method (HPM) [8-11]. The HPM is a combination of the classical perturbation technique and homotopy technique, firstly proposed by $\mathrm{He}$ [12-14]. One of the benefits of this method is that it hasn't Homotopy and perturbation methods limitation. In this paper, the basic HPM method presented and then problem of an incompressible viscous flow over a flat plate is presented and Blausis equation derived. Then analytical solution of problem is obtained by applying HPM method. In addition, Howarth number is calculated. Howarth number is an important number in fluid mechanics. It has used for calculating of drag coefficient. Howarth[15] obtained an accurate numerical solution for Blasius equation in which $\alpha=f^{\prime \prime}(0)=0.332057$. This value is accepted for comparison because it has high accuracy. After that, several try is done for calculating this number from other methods. He [16] in 1998 solved this equation by applying variational iteration method and he found $\alpha=0.5436$ by a first approximation. This value has $63.7 \%$ relative error respect to Howarth's calculation. In 2007 Wazwaz[17] used the same method and found $\alpha=0.37329$.This value has $21.42 \%$ relative error respect to Howarth. In 2003 and 2004, By homotopy method, $\mathrm{He}[18,19]$ obtained the first iteration step led to $\alpha=0.3095$ with $6.8 \%$ accuracy (relative error), and the second iteration step yielded $\alpha=0.3296$ with $0.7 \%$ accuracy of the initial slope. In 2007 Ganji [20] found $\alpha=0.348505$ by HPM method with three terms approximation with 4.9\% relative error. In 2009 Fathizadeh and Rashidi [21] found $\alpha=0.348$ by HPM method with $4.9 \%$ relative error. In this paper, the problem is solved by using a special technique and HPM method and then results are compared with previous works. The approximations of the $f^{\prime \prime}(0)$ obtained by this paper in comparison with previous HPM results provide the higher accuracy.

\section{Basic idea of Homotopy Perturbation Method}

To illustrate the basic ideas of this method, we consider the following equation

$A(u)-f(r)=0, r \in \Omega$

With the boundary condition of 
$B\left(u, \frac{\partial u}{\partial n}\right)=0, r \in \Gamma$

Where $A$ is a general differential operator, $B$ a boundary operator, $f(r)$ a known analytical function and $\Gamma$ is the boundary of the domain $\Omega$. A can be divided into two parts, which are $L$ and $N$, where $L$ is linear and $N$ is nonlinear. eq. (1) can therefore be rewritten as follows:

$L(u)+N(u)-f(r)=0, \quad r \in \Omega$

Homotopy perturbation structure is shown as follows

$H(v, p)=(1-p)\left[L(v)-L\left(u_{0}\right)\right]+p[A(v)-f(r)]=0$

Where

$v(r, p): \Omega \times[0,1] \rightarrow R$

In eq. (4), $p \in[0,1]$ is an embedding parameter and $u_{0}$ is the first approximation that satisfies the boundary condition. We can assume that the solution of eq. (4) can be written as a power series in ${ }^{p}$, as following

$v=v_{0}+p v_{1}+p^{2} v_{2}+\mathrm{L}=\sum_{i=0}^{n} v_{i} p^{i}$

And the best approximation for solution is

$u=\lim _{p \rightarrow 1} v=v_{0}+v_{1}+v_{2}+\mathrm{L}$

The above convergence is discussed in [22]. 


\section{Governing Equations:}

Boundary layer flow over a flat plate is governed by the continuity and the Navier-Stokes equations. Under the boundary layer assumptions, for a two dimensional, steady state, incompressible flow with zero pressure gradient over a flat plate, governing equations are simplified to:

$$
\begin{aligned}
& u \frac{\partial u}{\partial x}+v \frac{\partial u}{\partial y}=v \frac{\partial^{2} u}{\partial y^{2}} \\
& \frac{\partial u}{\partial x}+\frac{\partial v}{\partial y}=0
\end{aligned}
$$

Subjected to boundary conditions:

$$
\begin{aligned}
& \mathrm{y}=0 \rightarrow \mathrm{u}=0 ; \\
& y=\infty \rightarrow \mathrm{u}=\mathrm{U}_{\infty}, \frac{\partial u}{\partial y}=0
\end{aligned}
$$

By applying a dimensionless variable $\left({ }^{\eta}\right)$ defined as:

$$
\eta=\frac{y}{\sqrt{x}} \operatorname{Re}^{.5}
$$

( $\mathrm{Re}$ is the Reynolds number and defined as: $\left(\mathrm{Re}=\frac{\mathrm{U}_{\infty} x}{v}\right)$

The governing equations of (8) and (9) can be reduced to the well-known Blasius equation where $f$ is a function of variable $(\eta)$ :

$$
f^{\prime \prime \prime}+\frac{1}{2} f f^{\prime \prime}=0
$$


With boundary equations:

$$
\begin{aligned}
& \eta=0 \rightarrow f=0, f^{\prime}=0 \\
& \eta=\infty \rightarrow f^{\prime}=1
\end{aligned}
$$

Where $f$ is related to the $u$ velocity by

$$
f^{\prime}=\frac{u}{u_{\infty}}
$$

And the "prime" denotes the derivatives with respect to $\eta$.

\section{HPM Solution for Flow over a Flat Plate:}

For some sufficiently large $\mathrm{M}$, the condition (3) can be replaced by the condition

$$
f^{\prime}(M)=1
$$

Under the transformation $z=\frac{\eta}{M}$, the blasius equation transformed to:

$$
g^{\prime \prime \prime}(z)+\frac{M^{2}}{2} g(z) g^{\prime \prime}(z)=0
$$

Where $g(z)=\frac{f(\eta)}{M}$ and the "prime" denotes the derivatives with respect to $z \in[0,1]$.The boundary conditions (14), (15) are transformed to

$$
\begin{aligned}
& z=0 \rightarrow g=0, g^{\prime}=0 \\
& z=1 \rightarrow g^{\prime}=1
\end{aligned}
$$


In this section, we will apply the HPM to nonlinear ordinary differential system (18). According to the HPM, we can construct a homotopy of system (18) as follows:

$$
(1-p)\left(v^{\prime \prime \prime}-g_{0}^{\prime \prime \prime}\right)+p\left(v^{\prime \prime \prime}+\frac{M^{2}}{2} v v^{\prime \prime}\right)=0
$$

We consider $v$ as following:

$$
v=v_{0}+p v_{1}+p^{2} v_{2}+p^{3} v_{3}+\ldots=\sum_{i=0}^{6} v_{i} p^{i}
$$

Assuming $g_{0}^{\prime \prime \prime}=0$ and substituting $v$ from Eq. (22) into Eq. (21) and some simplification and rearranging based on powers of $p$-terms, we have:

$$
\begin{aligned}
& P^{\mathrm{O}}: v_{0}{ }^{\prime \prime \prime}=0 \\
& v_{0}(0)=0, v_{0}^{\prime}(0)=0, v_{0}^{\prime}(1)=1 \\
& P^{1}: v_{1}^{\prime \prime \prime}+\frac{M^{2}}{2}\left(v_{0} v_{0}^{\prime \prime}\right)=0 \\
& v_{1}(0)=0, v_{1}^{\prime}(0)=0, v_{1}^{\prime}(1)=0 \\
& P^{2}: v_{2}^{\prime \prime \prime}+\frac{M^{2}}{2}\left(v_{1} v_{0}^{\prime \prime}+v_{0} v_{1}^{\prime \prime}\right)=0 \\
& v_{2}(0)=0, v_{2}^{\prime}(0)=0, v_{2}^{\prime}(1)=0 \\
& P^{3}: v_{3}^{\prime \prime \prime}+\frac{M^{2}}{2}\left(v_{2} v_{0}^{\prime \prime}+v_{0}{ }_{2}^{\prime \prime}+v_{1} v_{1}^{\prime \prime}\right)=0 \\
& v_{3}(0)=0, v_{3}^{\prime}(0)=0, v_{3}^{\prime}(1)=0 \\
& P^{4}: v_{4}^{\prime \prime \prime}+\frac{M^{2}}{2}\left(v_{3} v_{0}^{\prime \prime}+v_{0}{ }^{\prime \prime} 3^{\prime \prime}+v_{2} v_{1}^{\prime \prime}+v_{1} v_{2}^{\prime \prime}\right)=0 \\
& v_{4}(0)=0, v_{4}^{\prime}(0)=0, v_{4}^{\prime}(1)=0 \\
& P^{5}: v_{5}^{\prime \prime \prime}+\frac{M^{2}}{2}\left(v_{3} v_{1}^{\prime \prime}+v_{1} v_{3}^{\prime \prime}+v_{4} v_{0}^{\prime \prime}+v_{0} v_{4}^{\prime \prime}+v_{2} v_{2}^{\prime \prime}\right)=0 \\
& v_{5}(0)=0, v_{5}^{\prime}(0)=0, v_{5}^{\prime}(1)=0
\end{aligned}
$$




$$
\begin{aligned}
& P^{6}: v_{6}^{\prime \prime \prime}+\frac{M^{2}}{2}\left(v_{4} v_{1}^{\prime \prime}+v_{1} v_{4}^{\prime \prime}+v_{5} 5_{0}^{\prime \prime}+v_{0}{ }^{v} 5^{\prime \prime}+v_{3} v_{2}{ }^{\prime \prime}++v_{2} v_{3}^{\prime \prime}\right)=0 \\
& v_{6}(0)=0, v_{6}^{\prime}(0)=0, v_{6}^{\prime}(1)=0
\end{aligned}
$$

Solving Eqs. (23) - (29) with boundary conditions, we have:

$$
\begin{aligned}
& v_{0}(z)=\frac{1}{2} z^{2} \\
& v_{1}(z)=\frac{-1}{240} M^{2} z^{5}+\frac{1}{96} M^{2} z^{2} \\
& v_{2}(z)=\frac{11}{161280} M^{4} z^{8}-\frac{1}{5760} M^{4} z^{5}+\frac{13}{80640} M^{4} z^{2} \\
& v_{3}(z)=\frac{-1}{107520} M^{6}\left(\frac{25}{198} z^{11}-\frac{11}{24} z^{8}+\frac{29}{60} z^{5}\right)+\frac{1}{1548288} M^{6} z^{2} \\
& v_{4}(z)=\frac{1}{425779200} M^{8}\left(\frac{9299}{1092} z^{14}-\frac{125}{3} z^{11}+\frac{7381}{112} z^{8}-\frac{341}{12} z^{5}\right)-\frac{50249}{929901772800} M^{8} z^{2} \\
& v_{5}(z)=\frac{-1}{1859803545600} M^{10}\left(\frac{1272379}{2040} z^{17}-\frac{46495}{12} z^{14}+\frac{51025}{6} z^{11}\right. \\
& \left.-\frac{162019}{24} z^{8}-\frac{27139}{60} z^{5}\right)-\frac{763571}{446352850944000} M^{10} z^{2} \\
& v_{6}(z)=\frac{1}{2529332822016000} M^{12}\left(\frac{19241647}{1368} z^{20}-\frac{1272379}{12} z^{17}+\frac{6323320}{21} z^{14}\right. \\
& \left.-\frac{3259750}{9} z^{11}+\frac{16488725}{168} z^{8}+\frac{6647629}{60} z^{5}\right)-\frac{59045561}{6055222775906304000} M^{12} z^{2}
\end{aligned}
$$

According to Eq. (22) and the assumption $p=1$ :

$$
g=\lim _{p \rightarrow 1} v=v_{0}+v_{1}+v_{2}+\mathrm{L}
$$

With choice $M=5$ we get: 


$$
\begin{aligned}
& g(z)=0.8310701384 z^{2}-0.2959136461 z^{5}+0.2146153977 z^{8}-0.1361900018 z^{11} \\
& +0.05722180311 z^{14}-0.01350962363 z^{17}+0.001357657484 z^{20}
\end{aligned}
$$

Now, under the transformation $g(z)=\frac{f(\eta)}{M}$, the above equation transformed to:

$$
\begin{aligned}
& f(\eta)=0.166214027 \eta^{2}-4.734618338 \times 10^{-4} \eta^{5}+2.747077090 \times 10^{-6} \eta^{8}-1.394585618 \times 10^{-8} \eta^{11} \\
& +4.687610111 \times 10^{-11} \eta^{14}-8.853666940 \times 10^{-14} \eta^{17}+7.118035270 \times 10^{-17} \eta^{20}
\end{aligned}
$$

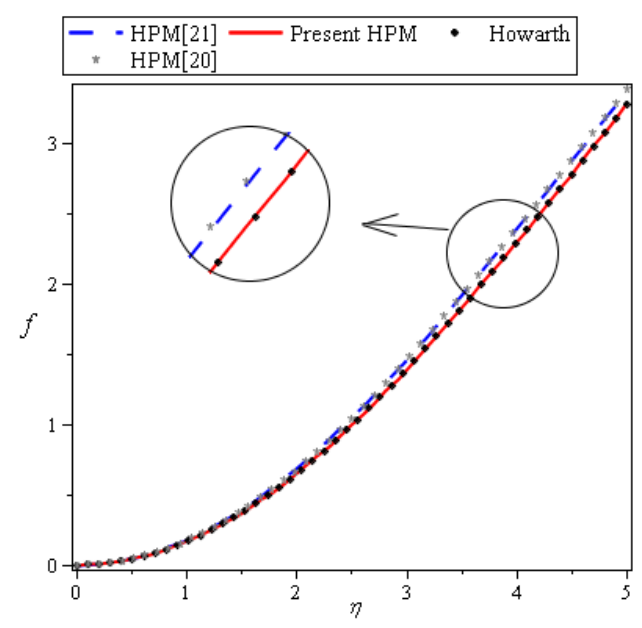

Fig. 1: The comparison of answers obtained by HPM and numerical solution for $f(\eta)$

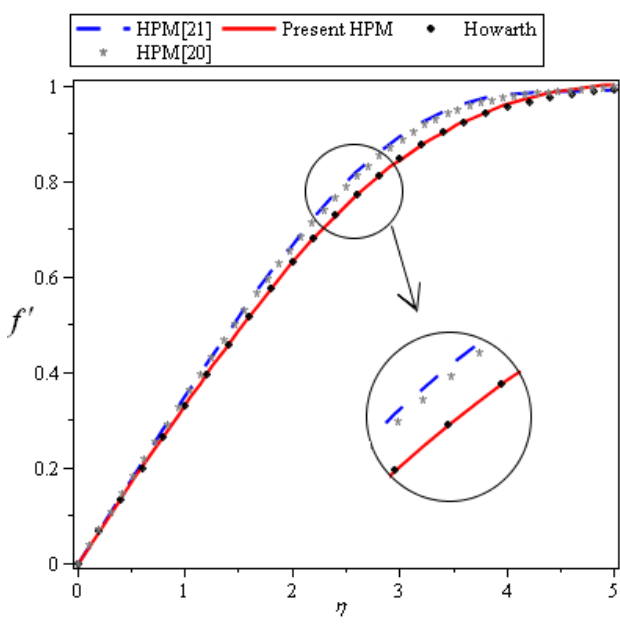

Fig. 2: The comparison of answers obtained by HPM and numerical solution for $f^{\prime}$

$(\eta)$

\section{Results and Discussion}

In this manuscript the Homotopy perturbation method such as analytical technique is employed for nonlinear blasius equation. Figs. 1 and 2 show the profiles of $f(\eta)$ and $f^{\prime}(\eta)$ obtained by the HPM for different values of $\eta$ in comparison with the numerical solutions obtained by Howarth. We can see a very good agreement between the present HPM and the numerical results. So, the solutions are obtained present HPM are more accurate than [20], [21]. Numerical comparison between present HPM with other different approximate solution is tabulated in Table 1 and 2. It is interesting to note that present HPM is very close to the numerical results in comparison with [20], [21]. The approximations of the $f^{\prime \prime}(0)$ obtained by HPM and their relative error with respect to the Howarth [15] results are listed in Table 
3. Figs. 3 and 4 shows the absolute error of $f, f^{\prime}$ related to Howarth solution at different values $\eta$. But our results are more acceptable than results obtained by [20], [21].

Table 1: Obtained results, in comparison with HPM and numerical method (Howarth) for $f(\eta)$

\begin{tabular}{|c|c|c|c|c|c|c|c|}
\hline$\eta$ & $\begin{array}{l}\text { Howarth } \\
\text { [19] }\end{array}$ & $\begin{array}{l}\text { Hpm } \\
{[20]}\end{array}$ & $\begin{array}{l}\text { Hpm } \\
{[21]}\end{array}$ & $\begin{array}{l}\text { Present } \\
\text { hpm }\end{array}$ & $\begin{array}{l}\text { Error } \\
\text { hpm } \\
{[20]}\end{array}$ & $\begin{array}{l}\text { Error } \\
\text { hpm } \\
{[21]}\end{array}$ & $\begin{array}{l}\text { Eroor } \\
\text { present }\end{array}$ \\
\hline 0 & 0 & 0 & 0 & 0 & 0 & 0 & 0 \\
\hline 0.2 & 0.0066412 & 0069699 & 0077932 & 0.0066484 & 0.0003287 & 0.0011520 & 0.0000072 \\
\hline 0.4 & 762 & 0278758 & 0293860 & 0.02658 & 0.0011996 & 0.0027098 & 0.0000868 \\
\hline 0.6 & 215 & 59 & 9647567 & 03 & 0.0029744 & 0.0050 & 788 \\
\hline 0.8 & 82 & 11137 & 1138493 & 24 & 0.0052656 & 0.0077 & \\
\hline 1.0 & & & & 0.1657 & 0.0082299 & $0.010 s$ & \\
\hline 1.2 & & & & & 0.0118551 & 0.0147542 & \\
\hline 1.4 & & & & & 0.0161 & 0.0190 & \\
\hline 1.6 & & & & & 0.0210801 & $0.023 \varepsilon$ & \\
\hline 1.8 & & & & & 0.0266617 & 0.029 & \\
\hline 2.0 & & & & & 0.0328590 & 0.035 & \\
\hline 2.2 & & & & & 0.0396273 & 0.0412 & \\
\hline 2.4 & & & & & 0.0468972 & 0.0478580 & 0.0002238 \\
\hline 2.6 & & 1.1270772 & & & 0.0545713 & 0.0547 & 0.0001011 \\
\hline 2.8 & 1.2309773 & 1.2935005 & 1.2926472 & 1.2309370 & 0.0625232 & 0.0616 & 0.0000403 \\
\hline 3.0 & 1.3968082 & 1.4674133 & 1.4653338 & 1.3966410 & 0.0706051 & 0.0685 & 0.0001672 \\
\hline 3.2 & 1.5690949 & 1.6477584 & 1.6441417 & 1.5688630 & 0.0786635 & 0.0750468 & 0.0002319 \\
\hline 3.4 & 1.7469501 & 1.8335195 & 1.8279185 & 1.7467840 & 0.0865694 & 0.0809684 & 0.0001661 \\
\hline 3.6 & 1.9295251 & 2.0237911 & 2.0155409 & 1.9296270 & 0.0942660 & 0.0860158 & 0.0001019 \\
\hline 3.8 & 2.1160298 & 2.2178650 & 2.2059613 & 2.1166770 & 0.1018352 & 0.0899315 & 0.0006472 \\
\hline 4.0 & 2.3057464 & 2.4153361 & 2.3982576 & 2.3072780 & 0.1095897 & 0.0925112 & 0.0015316 \\
\hline 4.2 & 2.4980396 & 2.6162294 & 2.5916832 & 2.5008250 & 0.1181898 & 0.0936436 & 0.0027854 \\
\hline 4.4 & 2.6923609 & 2.8211494 & 2.7857122 & 2.6967390 & 0.1287885 & 0.0933513 & 0.0043781 \\
\hline 4.6 & 2.8882480 & 3.0314545 & 2.9800744 & 2.8944690 & 0.1432065 & 0.0918264 & 0.0062210 \\
\hline 4.8 & 3.0853206 & 3.2494582 & 3.1747721 & 3.0934800 & 0.1641376 & 0.0894515 & 0.0081594 \\
\hline 5.0 & 3.2832736 & 3.4786579 & 3.3700690 & 3.2932580 & 0.1953843 & 0.0867954 & 0.0099844 \\
\hline
\end{tabular}

Table 2: Obtained results for $f^{\prime \prime}(0)$, in comparison with HPM and numerical method, Howarth $\left(f^{\prime \prime}(0)=.332057\right)$

\begin{tabular}{|l|l|l|}
\hline method & $f^{\prime \prime}(0)$ & Relative Error \\
\hline HPM [Present method] & 0.332428 & $0.1 \%$ \\
\hline HPM [20] & 0.348505 & $4.9 \%$ \\
\hline HPM [21] & 0.348 & $4.8 \%$ \\
\hline HPM [18] & 0.3095 & $6.8 \%$ \\
\hline HAM[19] & 0.3296 & $0.7 \%$ \\
\hline
\end{tabular}


Table 3: Obtained results, in comparison with HPM and numerical method (Howarth) for $f^{\prime}(\eta)$

\begin{tabular}{|c|c|c|c|c|c|c|c|}
\hline$\eta$ & $\begin{array}{l}\text { Howarth } \\
{[19]}\end{array}$ & $\begin{array}{l}\text { Hpm } \\
{[20]}\end{array}$ & $\begin{array}{l}\mathrm{Hpm} \\
{[21]}\end{array}$ & $\begin{array}{l}\text { Present } \\
\text { hpm }\end{array}$ & $\begin{array}{l}\text { Error } \\
\text { HPM } \\
{[20]}\end{array}$ & $\begin{array}{l}\text { Error } \\
\text { HPM } \\
{[21]}\end{array}$ & $\begin{array}{l}\text { Error } \\
\text { present }\end{array}$ \\
\hline 0 & 0 & 0 & 0 & 0 & 0 & 0 & 0 \\
\hline 0.2 & 0.0664077 & 0.0696975 & 0.0703281 & 0.0664818 & 0.0032898 & 0.0039204 & 0.0000741 \\
\hline 0.4 & 0.1327641 & 0.1393444 & 0.1406060 & 0.1329106 & 0.0065803 & 0.0078419 & 0.0001465 \\
\hline 0.6 & 0.19 & 0.2088105 & 0.2107046 & 1507 & 0.0098733 & 0.0117674 & 135 \\
\hline 0.8 & 0.2647094 & 0.2778800 & 0.2804100 & 0.2649775 & 0.0131706 & 0.0157006 & 0.00 \\
\hline 1.0 & 0.329 & 0.346 & 0.3494253 & 0.3300826 & 0.0164738 & 0.0196453 & \\
\hline 1.2 & 0.393 & 0.413 & 0.4173749 & 0.3940826 & 0.0197778 & 0.0235 & 3065 \\
\hline 1.4 & 0.4562617 & 0.4793309 & 0.4838112 & 0.4565324 & 0.0230692 & 0.0275495 & 0.0002707 \\
\hline 1.6 & 0.5167567 & 0.5430747 & 0.5482248 & 0.5169439 & 0.0263180 & 0.0314681 & 0.0001872 \\
\hline 1.8 & 0.5747581 & 0.6042289 & 0.6100571 & & 0.0294708 & 0.0352990 & \\
\hline 2.0 & 0.62 & 0.662 & 0.6687189 & & 0.0324440 & 0.038 & \\
\hline 2.2 & 0.6813103 & 0.7164291 & 0.7236108 & 0.6809787 & 0.0351188 & 0.0423005 & 0.0003316 \\
\hline 2.4 & 0.72 & 0.766 & 0.77 & 4483 & 0.037 & 0.04 & \\
\hline 2.6 & 0.7724550 & 0.8113803 & 0.8197988 & 0.7717748 & 0.0389253 & 0.0473438 & 0.0006802 \\
\hline 2.8 & 0.8115096 & 0.8511819 & 0.8600992 & 0.8108053 & 0.0396723 & 0.0485896 & 0.0007043 \\
\hline 3.0 & 0.8460444 & 0.8854328 & 0.8947068 & 0.8455199 & 0.0393884 & 0.0486624 & 0.0005245 \\
\hline 3.2 & 0.8760814 & 0.9140010 & 0.9234279 & & 0.0379196 & 0.0473465 & 0.0000579 \\
\hline 3.4 & 0.901 & 0.9369507 & 0.9462547 & 0.9025271 & 0.0351 & 0.0444935 & 0.0007659 \\
\hline 3.6 & 0.9233296 & 0.9545718 & 0.9633968 & 0.9253044 & 0.0312422 & 0.0400672 & 0.0019748 \\
\hline 3.8 & 0.9411181 & 0.9673977 & 0.9753066 & 0.9446574 & 0.0262796 & 0.0341885 & 0.0035393 \\
\hline 4.0 & 0.9555182 & 0.9762106 & 0.9826929 & 0.9608570 & 0.0206924 & 0.0271747 & 0.0053388 \\
\hline 4.2 & 0.9669570 & 0.9820237 & 0.9865191 & 0.9741165 & 0.0150667 & 0.0195621 & 0.0071595 \\
\hline 4.4 & 0.9758708 & 0.9860369 & 0.9879789 & 0.9845695 & 0.0101661 & 0.0121081 & 0.0086987 \\
\hline 4.6 & 0.9826835 & 0.9895542 & 0.9884434 & 0.9922967 & 0.0068707 & 0.0057599 & 0.0096132 \\
\hline 4.8 & 0.9877895 & 0.9938540 & 0.9893700 & 0.9973820 & 0.0060645 & 0.0015805 & 0.0095925 \\
\hline 5.0 & 0.9915419 & 0.9999999 & 0.9921642 & 0.9999982 & 0.0084580 & 0.0006223 & 0.0084563 \\
\hline
\end{tabular}

\section{Conclusion}

In this paper, the Homotopy perturbation method has been successfully applied to a nonlinear blasius problem. Since we have used a special technique, we have obtained results with excellent accuracy for $\eta \leq 5$. It is also shown that, the present

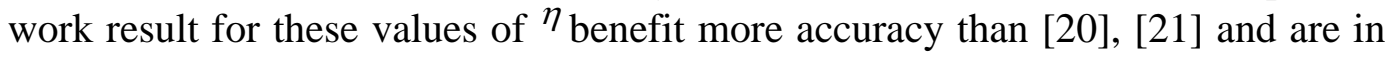
acceptable agreement with ones derived by the numerical method. 


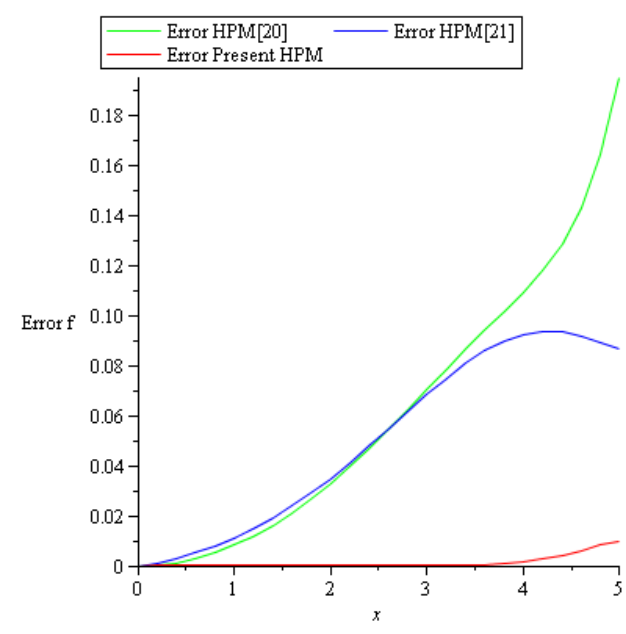

Fig. 3: The comparison of the absolute error in answers results by HPM for $f(\eta)$ with respect to the Howarth [15] results

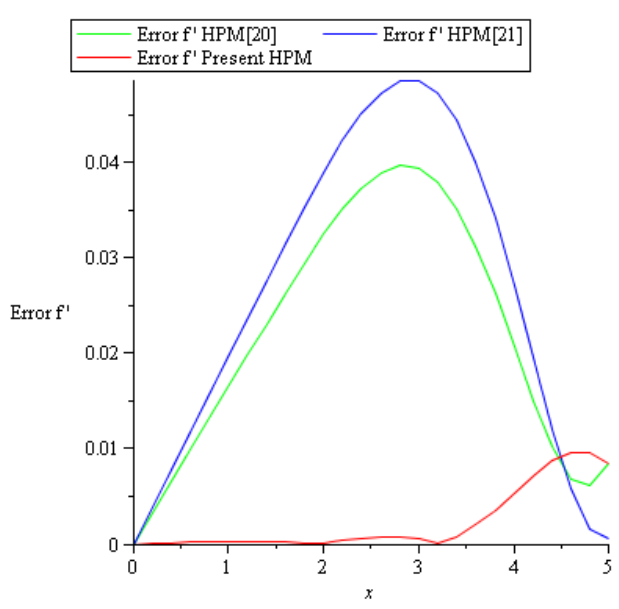

Fig. 4: The comparison of the absolute error in answers results by HPM for $f^{\prime}(\eta)$ with respect to the Howarth [15] results

\section{References}

[1] A.H. Nayfeh, Perturbation Methods, Wiley, New York, 2000.

[2] J.D. Cole, Perturbation Methods in Applied Mathematics, Blaisdell Waltham, MA, 1968.

[3] A.V. Karmishin, A.I. Zhukov, V.G. Kolosov, Methods of Dynamics Calculation and Testing for Thin-Walled Structures, Mashinostroyenie, Moscow, 1990.

[4] A.M. Lyapunov, General Problem on Stability of Motion, Taylor \& Francis, London, 1992 (English translation).

[5] J. Biazar, E. Babolian, R. Islam, Solution of a system of Volterra integral equations of the first kind by Adomian method, Journal of Applied Mathematics and Computation 139 (2003)), pp. 249-258.

[6] S.Q. Wang, J.H. He, Variational iteration method for solving integrodifferential equations, Journal of Physics 367 (2007) ), pp. 188-191.

[7] M.M. Rashidi, G. Domairry, S. Dinarvand, Approximate solutions for the Burger and regularized long wave equations by means of the Homotopy analysis method, Communications in Nonlinear and Numerical Simulation 14 (2009)), pp. 708-717.

[8] [8] J.H. He, Homotopy perturbation method for bifurcation on nonlinear problems, International Journal of Non-linear Science and Numerical Simulation 6 (2005) ), pp. 207-218. 
[9] J.H. He, New interpretation of homotopy perturbation method, International Journal of Modern Physics B 20 (2006) ), pp. 2561-2568.

[10] D.D. Ganji, A. Rajabi, Assessment of homotopy-perturbation and perturbation methods in heat radiation equations, Int. Commun. Heat Mass Transfer 33 (2006)), pp. 391-400.

[11] D.D. Ganji, M. Rafei, Explicit solutions of Helmholtz equation and fifthorder KDV equation using homotopy perturbation method, Int. J. Non-linear Sci. Numer. Simul. 7 (2006) ), pp. 321-329.

[12] J.H. He, Homotopy perturbation method: A new nonlinear analytical technique, Applied Mathematics and Computation 135 (2003) ), pp. 73-79.

[13] J.H. He, Homotopy perturbation method for solving boundary value problems, Physics Letters A 350 (2006) ), pp. 87-88.

[14] J.H. He, The homotopy perturbation method for nonlinear oscillators with discontinuities, Applied Mathematics and Computation 15 (2004) ), pp. 287292.

[15] L. Howarth, On the calculation of steady flow in the boundary layer near the surface of a cylinder in a stream, Aero Res. Counc. Lond. R \&M164 (1935) ), pp.16-32.

[16] J.H. He, Approximate Analytical Solution of Blasius Equation, Non-linear Science Numerical Simulation 3 (1998) ), pp. 260-263.

[17] A.M. Wazwaz, The Variational Iteration Method for Solving Two Forms of Blasius Equation on a Half Infinite Domain, Applied Mathematics and Computation 188 (2007) ), pp. 485-491.

[18] J.H. He, A Simple Perturbation Approach to Blasius Equation, Applied Mathematics and Computation 140 (2003) ), pp. 217-222.

[19] J.H. He, Comparison of Homotopy Perturbation Method and Homotopy Analysis Method,Applied Mathematics and Computation, 156 (2004)), pp. 527-539.

[20] M. Esmaeilpour, D.D. Ganji, Application of He's Homotopy perturbation method to boundary layer flow and convection heat transfer over a flat plate, Physics Letters A 372 (2007) ), pp. 33-38.

[21] M. Fathizadeh, F. Rashidi, Boundary layer convective heat transfer with pressure gradient using Homotopy Perturbation Method (HPM) over a flat plate, Chaos, Solitons and Fractals 42 (2009) ), pp. 2413-2419.

[22] S.H. Hosein Nia, A.N. Ranjbar, D.D. Ganji, H. Soltani, J. Ghasemi, Maintaining the stability of nonlinear differential equations by the enhancement of HPM, Physics Letters A 372 (16) (2008), pp. 2855-2861. 